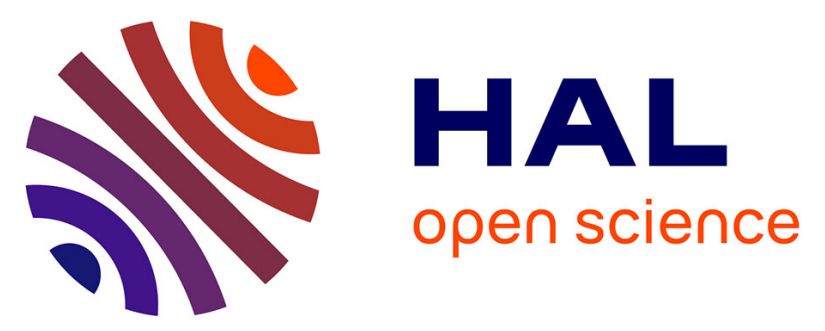

\title{
Boundary Observers for Linear and Quasi-linear Hyperbolic Systems with Application to Flow Control
}

Felipe Castillo Buenaventura, Emmanuel Witrant, Christophe Prieur, Luc Dugard

\section{- To cite this version:}

Felipe Castillo Buenaventura, Emmanuel Witrant, Christophe Prieur, Luc Dugard. Boundary Observers for Linear and Quasi-linear Hyperbolic Systems with Application to Flow Control. Automatica, 2013, 49 (11), pp.3180-3188. 10.1016/j.automatica.2013.07.027 . hal-00841207

\section{HAL Id: hal-00841207 \\ https://hal.science/hal-00841207}

Submitted on 17 Jul 2013

HAL is a multi-disciplinary open access archive for the deposit and dissemination of scientific research documents, whether they are published or not. The documents may come from teaching and research institutions in France or abroad, or from public or private research centers.
L'archive ouverte pluridisciplinaire HAL, est destinée au dépôt et à la diffusion de documents scientifiques de niveau recherche, publiés ou non, émanant des établissements d'enseignement et de recherche français ou étrangers, des laboratoires publics ou privés. 


\title{
Boundary Observers for Linear and Quasi-Linear Hyperbolic Systems with Application to Flow Control
}

\author{
Felipe Castillo $^{\text {a }}$, Emmanuel Witrant ${ }^{\mathrm{a}}$, Christophe Prieur ${ }^{\mathrm{a}}$, Luc Dugard ${ }^{\mathrm{a}}$ \\ ${ }^{\mathrm{a}}$ GIPSA-Lab, 11 rue des mathématiques, BP 46, 38402 Saint Martin d'Hères Cedex, France
}

\begin{abstract}
In this paper we consider the problem of boundary observer design for one-dimensional first order linear and quasi-linear strict hyperbolic systems with $n$ rightward convecting transport PDEs. By means of Lyapunov based techniques, we derive some sufficient conditions for exponential boundary observer design using only the information from the boundary control and the boundary conditions. We consider static as well as dynamic boundary controls for the boundary observer design. The main results are illustrated on the model of an inviscid incompressible flow.
\end{abstract}

Key words: Boundary observers; hyperbolic systems; infinite dimensional observer.

\section{Introduction}

Techniques based on Lyapunov functions are commonly used for the stability analysis of infinite dimensional dynamical systems, such as those described by strict hyperbolic partial differential equations (PDE). Many distributed physical systems are described by such models. For example, the conservation laws describing process evolution in open conservative systems are described by hyperbolic PDEs. One of the main properties of this class of PDE is the existence of the so-called Riemann transformation which is a powerful tool for the proof of classical solutions, analysis and control, among other properties [2]. Among the potential applications, hydraulic networks [17], multiphase flow [14], road traffic networks [9], gas flow in pipelines [3] or flow regulation in deep pits [21] are of significant importance. The interest on boundary observers comes from the fact that measurements in distributed parameter systems are usually not available. It is more common for sensors to be located at the boundaries.

Control results for first-order hyperbolic systems do exist in the literature. In [13], sufficient conditions on the structure of the control problem for controlability and

Email addresses:

felipe.castillo-buenaventura@gipsa-lab.fr (Felipe Castillo), emmanuel.witrant@ujf-grenoble.fr (Emmanuel Witrant), christophe.prieur@gipsa-lab.fr (Christophe Prieur), luc.dugard@gipsa-lab.fr (Luc Dugard). observability of such systems are given. The stability problem of the boundary control in hyperbolic systems has been exhaustively investigated, e.g. in [5] [6] [7] [11] [15], among other references. However, boundary observers for hyperbolic systems have been less explored. The boundary observability of infinite dimensional linear systems has been discussed in [19] with operator semigroups acting on Hilbert spaces. In [12], backstepping boundary observer design for linear PDE's has been introduced where the observer gains are found by solving a supplementary set of PDEs. Some of the most recent results on observation of first order hyperbolic systems can be found in [1], where exponential convergence has been shown by Lyapunov method, for linearized hyperbolic models using boundary injections. In [20], the problem of boundary stabilization and state estimation for a $2 \times 2$ system of first order hyperbolic linear PDEs with spatially varying coefficients is considered. In [4], discrete approximations of this kind of systems have been used to address the observation problem when having dynamics associated with the boundary control. Nevertheless, a generalization for the boundary observer design of linear and quasi-linear onedimensional conservation laws with static and dynamic boundary control has not been found in the literature.

Let $n$ be a positive integer and $\Theta$ be an open non-empty convex set of $\mathbb{R}^{n}$. In this work we consider the following class of quasi-linear hyperbolic systems of order $n$ : 


$$
\partial_{t} \xi(x, t)+\Lambda(\xi) \partial_{x} \xi(x, t)=0 \quad \forall x \in[0,1], t \geq 0
$$

where $\xi:[0,1] \times[0, \infty) \rightarrow \mathbb{R}^{n}$ and $\Lambda$ is a continuously differentiable diagonal matrix function $\Lambda: \Theta \rightarrow \mathbb{R}^{n \times n}$ such that $\Lambda(\xi)=\operatorname{diag}\left(\lambda_{1}(\xi), \lambda_{2}(\xi), \ldots, \lambda_{n}(\xi)\right)$. Let us assume the following:

Assumption 1: The following inequalities hold:

$$
0<\lambda_{1}(\xi)<\lambda_{2}(\xi)<\ldots<\lambda_{n}(\xi) \quad \forall \xi \in \Theta
$$

If $\Lambda(\xi)=\Lambda$, then (1) is a linear hyperbolic system given by:

$$
\partial_{t} \xi(x, t)+\Lambda \partial_{x} \xi(x, t)=0 \quad \forall x \in[0,1], t \geq 0
$$

We consider two types of boundary control for the quasilinear hyperbolic system (1). The first one is a static boundary control given by:

$$
\xi(0, t)=u_{c}(t) \quad \forall t \geq 0
$$

and the second one is a dynamic boundary control:

$$
\begin{aligned}
& \dot{X}_{c}(t)=A X_{c}(t)+B u_{c}(t) \\
& \xi(0, t)=C X_{c}(t)+D u_{c}(t) \quad \forall t \geq 0
\end{aligned}
$$

where $X_{c}(t) \in \mathbb{R}^{n}, A \in \mathbb{R}^{n \times n}, B \in \mathbb{R}^{n \times n}, C \in \mathbb{R}^{n \times n}$, $D \in \mathbb{R}^{n \times n}$ and $u_{c} \in C^{1}\left([0, \infty), \mathbb{R}^{n}\right)$. The initial condition is defined as:

$$
\begin{gathered}
\xi(x, 0)=\xi^{0}(x), \quad \forall x \in[0,1] \\
X_{c}(0)=X_{c}^{0}
\end{gathered}
$$

Remark 1: The initial condition of (1) with static boundary conditions (4) is given by (6).
It has been proved, (see e.g. [6] and [10] among other references), that there exist a $\delta_{0}>0$ and a $T>0$ such that for every $\xi^{0} \in H^{2}\left((0,1), \mathbb{R}^{n}\right)$ satisfying $\left|\xi^{0}\right|_{H^{2}\left((0,1), \mathbb{R}^{n}\right)}<$ $\delta_{0}$ and the zero-order and one-order compatibility conditions, the Cauchy problem ((1), (4) and (6)) and ((1), $(5),(6)$ and (7)) has a unique maximal classical solution satisfying:

$$
|\xi(., t)|_{H^{2}}<\delta_{0} \quad \forall t \in[0, T)
$$

Moreover, for linear hyperbolic systems (3), it holds for $T=+\infty$. For the quasi-linear hyperbolic system (1), the following assumption is necessary for some of the results considered later:

Assumption 2: Given a sufficiently small initial condition (6), the solutions for (1), with boundary condition (4) or (5) and initial condition (6) are assumed to be defined for all $t>0$.

Remark 2: Under Assumption 1 and the boundary conditions (4), there is no coupling between the states and thus an observer can be designed for each state separately. However, this is not true for the dynamic boundary conditions (5) as it induces a coupling between the states and motivates further analysis for the observer design.

Our main contribution is to develop sufficient conditions for infinite dimensional boundary observers design for linear and quasi-linear strict hyperbolic systems with $n$ rightward convecting PDEs in presence of static (4) as well as dynamic boundary control (5). To demonstrate the asymptotic convergence of the estimation error, a strict Lyapunov function formulation is used. The sufficient conditions are derived in terms of the system's and boundary conditions dynamics. In Proposition 1 and Theorem 1, we present the sufficient conditions for the observer design for linear hyperbolic systems with static and dynamic boundary control, respectively, $\forall \xi^{0}:[0,1] \rightarrow \Theta$. Then, in Theorems 2 and 3 , some sufficient conditions for boundary observer design for quasi-linear hyperbolic systems are determined for $\xi^{0}:[0,1] \rightarrow \Upsilon \subset \Theta$ (the subset $\Upsilon$ is defined in details in Section 4). Finally, in Section 5, we present some of the main results applied to a flow speed boundary observer for two inviscid incompressible flows coupled by the boundary conditions.

Notation. By the expression $H \succeq 0$ and $H \preceq 0$ we mean that the matrix $H$ is a positive semi-definite and a negative semi-definite matrix, respectively. $H \succ 0$ and $H \prec 0$ stand for positive definite and negative definite, 
respectively. The usual Euclidian norm in $\mathbb{R}^{n}$ is denoted by $|$.$| and the associated matrix norm is denoted \|$.$\| .$ Given $\gamma>0, B(\gamma)$ is the open ball centered in 0 with radius $\gamma$. Given $g:[0,1] \rightarrow \mathbb{R}^{n}$, we define its $L^{2}$-norm (when is finite) as:

$$
\|g\|_{L^{2}}=\sqrt{\int_{0}^{1}|g(x)|^{2} d x}
$$

The $H^{1}$-norm of $g$ is given by $\|g\|_{H^{1}}=\|g\|_{L^{2}}+\left\|\partial_{x} g\right\|_{L^{2}}$ and the $L^{\infty}$-norm of $g$ is defined as:

$$
\|g\|_{L^{\infty}}=\sup _{x \in(0,1)}\{|g(x)|\}
$$

\section{Problem Formulation}

We consider the problem of establishing a Lyapunov approach to solve the problem of finding a state estimate $\hat{\xi}$ of $\xi$ from the knowledge of the boundary control $u_{c}(t)$ and $\xi(1, t)$. More specifically, we focus on the design of exponential boundary observers defined as follows:

Definition 1: Consider, for all $x \in[0,1]$ and $t \geq 0$, the boundary observer given by the following system:

$$
\partial_{t} \hat{\xi}(x, t)+\Lambda(\hat{\xi}) \partial_{x} \hat{\xi}(x, t)=0
$$

with the boundary conditions:

$$
\begin{aligned}
& \dot{X}_{o}(t)=f\left(X_{o}(t), u_{c}(t), v(t)\right) \\
& \hat{\xi}(0, t)=h\left(X_{o}(t), u_{c}(t), v(t)\right)
\end{aligned}
$$

where $v(t) \in \mathbb{R}^{n}$ is the observer input, $X_{o}(t) \in \mathbb{R}^{n}, f$ : $\mathbb{R}^{n} \times \mathbb{R}^{n} \times \mathbb{R}^{n} \rightarrow \mathbb{R}^{n}$ and $h: \mathbb{R}^{n} \times \mathbb{R}^{n} \times \mathbb{R}^{n} \rightarrow \mathbb{R}^{n}$. The initial condition is:

$$
\hat{\xi}(x, 0)=\hat{\xi}^{0}(x), \quad X_{o}(0)=X_{o}^{0}
$$

If there exist $M_{0}>0$ and $\alpha_{0}>0$ such that for all $\xi$ (solution of (1), (4) and (6) or (1), (5) and (6)) and $\hat{\xi}$ (solution of (9), (10) and (11)) the inequality

$$
\begin{aligned}
& \left\|X_{c}(t)-X_{o}(t)\right\|^{2}+\|\hat{\xi}(., t)-\xi(., t)\|_{L^{2}}^{2} \\
& \leq M_{0} e^{-\alpha_{0} t}\left(\left\|X_{c}(0)-X_{o}(0)\right\|^{2}+\left\|\hat{\xi}^{0}-\xi^{0}\right\|_{L^{2}}^{2}\right), \forall t \geq 0
\end{aligned}
$$

holds, then (9) with boundary conditions (10) and initial condition (11) is called an exponential boundary observer.

We dedicate the following two sections to the design of exponential boundary observer design for linear and quasi-linear hyperbolic systems with static boundary control (4) and dynamic boundary control (5).

\section{Boundary Observer for Linear Hyperbolic Systems}

The first problem we solve is the boundary observer design for (3) and (6) with static boundary conditions (4). The following proposition presents some sufficient conditions for this boundary observer design:

Proposition 1. Consider the system (3) with static boundary conditions (4) and initial condition (6). Let $P \in \mathbb{R}^{n \times n}$ be a diagonal positive definite matrix, $\mu>0$ be a constant and $L \in \mathbb{R}^{n \times n}$ be an observer gain such that:

$$
e^{-\mu} \Lambda P-L^{T} \Lambda P L \succeq 0
$$

then:

$$
\begin{aligned}
& \partial_{t} \hat{\xi}(x, t)+\Lambda \partial_{x} \hat{\xi}(x, t)=0 \\
& \hat{\xi}(0, t)=u_{c}(t)+L(\xi(1, t)-\hat{\xi}(1, t))
\end{aligned}
$$

is an exponential boundary observer for all twice continuously differentiable functions $\hat{\xi}^{0}:[0,1] \rightarrow \Theta$ satisfying the zero-order and one-order compatibility conditions.

Proof Define the estimation error $\varepsilon=\xi-\hat{\xi}$ whose dynamics is given by:

$$
\partial_{t} \varepsilon(x, t)+\Lambda \partial_{x} \varepsilon(x, t)=0
$$




$$
\varepsilon(0, t)=-L(\xi(1, t)-\hat{\xi}(1, t))=-L \varepsilon(1, t)
$$

The problem of the exponential convergence of (16) with boundary conditions (17) has been already considered in [6], However, we develop the proof for illustrating purposes, the sake of completeness and to allow the discussion of speed convergence. Given a diagonal positive definite matrix $P$, consider the quadratic Lyapunov function candidate proposed by [7] and defined for all continuously differentiable functions $\varepsilon:[0,1] \rightarrow \Theta$ as:

$$
V(\varepsilon)=\int_{0}^{1}\left(\varepsilon^{T} P \varepsilon\right) e^{-\mu x} d x
$$

where $\mu$ is a positive scalar. Computing the time derivative $\dot{V}$ of $V$ along the classical $C^{1}$-solutions of (16) with boundary conditions (17) yields to the following (after integrating by parts):

$$
\dot{V}=-\left.\left[e^{-\mu x} \varepsilon^{T} \Lambda P \varepsilon\right]\right|_{0} ^{1}-\mu \int_{0}^{1}\left(\varepsilon^{T} \Lambda P \varepsilon\right) e^{-\mu x} d x
$$

The boundary conditions (17) imply that:

$$
\begin{aligned}
\dot{V}= & -\varepsilon^{T}(1)\left[e^{-\mu} \Lambda P-L^{T} \Lambda P L\right] \varepsilon(1) \\
& -\mu \int_{0}^{1}\left(\varepsilon^{T} \Lambda P \varepsilon\right) e^{-\mu x} d x
\end{aligned}
$$

where $\varepsilon(1)=\varepsilon(1, t)$. For a positive small enough $\mu$ and (13), the first term of (20) is always negative or zero. From (2) it can be proved that there always exists an $\varrho>0$ such that $\Lambda>\varrho I^{n \times n}$ (e.g $\varrho$ could be the smallest eigenvalue of $\Lambda$ ). Moreover, the diagonality of $P$ and $\Lambda$ implies that:

$$
\dot{V} \leq-\mu \varrho V(\varepsilon)
$$

Therefore, the function (18) is a Lyapunov function for the hyperbolic system (16) with boundary conditions (17). This concludes the proof.
Note that (13) and (21) imply that $\mu$ is a part of the observer design as it explicitly enables to design the convergence speed. As the value of $\mu$ increases, the smaller the observer gain $L$ has to be in order to satisfy (13). Having an observer gain $L=0$ gives a trivial solution for Proposition 1. However, the boundary observer parameters $\mu$ and $L$ allow the observer performance design.

The second problem we consider is the boundary observer design for (3) and (6) with dynamic boundary conditions (5). This is solved with the following theorem.

Theorem 1. Consider the system (3) with dynamic boundary conditions (5) and initial condition (6)-(7). Assume that there exist two diagonal positive definite matrices $P_{1}, P_{2} \in \mathbb{R}^{n \times n}$, a constant $\mu>0$ and an observer gain $L \in \mathbb{R}^{n \times n}$ such that:

$$
\left[\begin{array}{cc}
A^{T} P_{1}+P_{1} A+C^{T} \Lambda P_{2} C+\mu \Lambda P_{1} & -P_{1} L \\
-L^{T} P_{1} & -e^{-\mu} \Lambda P_{2}
\end{array}\right] \preceq 0
$$

then:

$$
\partial_{t} \hat{\xi}(x, t)+\Lambda \partial_{x} \hat{\xi}(x, t)=0
$$

$$
\begin{aligned}
& \dot{\hat{X}}_{c}=A \hat{X}_{c}+B u_{c}(t)+L(\xi(1, t)-\hat{\xi}(1, t)) \\
& \hat{\xi}(0, t)=C \hat{X}_{c}+D u_{c}(t)
\end{aligned}
$$

is an exponential boundary observer for all twice continuously differentiable functions $\hat{\xi}^{0}:[0,1] \rightarrow \Theta$ and for all $\hat{X}_{c}^{0} \in \mathbb{R}^{n}$ satisfying the zero-order and one-order compatibility conditions.

Proof Define the dynamics of the estimation error $\varepsilon=$ $\xi-\hat{\xi}$ as follows:

$$
\partial_{t} \varepsilon(x, t)+\Lambda \partial_{x} \varepsilon(x, t)=0
$$

with boundary conditions: 


$$
\begin{aligned}
& \dot{\varepsilon}_{c}=A \varepsilon_{c}-L \varepsilon(1, t) \\
& \varepsilon(0, t)=C \varepsilon_{c}
\end{aligned}
$$

where $\varepsilon_{c}=X_{c}-\hat{X}_{c}$. Given the diagonal positive definite matrices $P_{1}$ and $P_{2}$, consider, as an extension of the Lyapunov function proposed in [7], the quadratic Lyapunov function candidate defined for all continuously differentiable functions $\varepsilon:[0,1] \rightarrow \Theta$ as:

$$
V\left(\varepsilon, \varepsilon_{c}\right)=\varepsilon_{c}^{T} P_{1} \varepsilon_{c}+\int_{0}^{1}\left(\varepsilon^{T} P_{2} \varepsilon\right) e^{-\mu x} d x
$$

Note that (28) has some similarities with respect to the Lyapunov function proposed in [16] for boundary control with integral action. Computing the time derivative $\dot{V}$ of $V$ along the classical $C^{1}$-solutions of (25) with boundary conditions (26) yields to the following:

$$
\begin{aligned}
\dot{V}= & \varepsilon_{c}^{T}\left(A^{T} P_{1}+P_{1} A\right) \varepsilon_{c}-\varepsilon(1)^{T} L^{T} P_{1} \varepsilon_{c}-\varepsilon_{c}^{T} P_{1} L \varepsilon(1) \\
& -\left.\left[e^{-\mu x} \varepsilon^{T} \Lambda P_{2} \varepsilon\right]\right|_{0} ^{1}-\mu \int_{0}^{1}\left(\varepsilon^{T} \Lambda P_{2} \varepsilon\right) e^{-\mu x} d x
\end{aligned}
$$

which can be written in terms of the boundary conditions as follows:

$$
\begin{aligned}
\dot{V}= & -\mu \varepsilon_{c}^{T} \Lambda P_{1} \varepsilon_{c}-\mu \int_{0}^{1}\left(\varepsilon^{T} \Lambda P_{2} \varepsilon\right) e^{-\mu x} d x+\left[\begin{array}{c}
\varepsilon_{c} \\
\varepsilon(1)
\end{array}\right]^{T} \\
& \times\left[\begin{array}{cc}
A^{T} P_{1}+P_{1} A+C^{T} \Lambda P_{2} C+\mu \Lambda P_{1} & -P_{1} L \\
-L^{T} P_{1} & -e^{-\mu} \Lambda P_{2}
\end{array}\right] \\
& \times\left[\begin{array}{c}
\varepsilon_{c} \\
\varepsilon(1)
\end{array}\right]
\end{aligned}
$$

Note that (22) implies that the third term of (30) is always negative or zero. Using the same procedure as in the proof of Proposition 1, it can be easily shown that there exists an $\varrho>0$ such that:

$$
\dot{V} \leq-\mu \varrho V\left(\varepsilon, \varepsilon_{c}\right)
$$

Therefore, the function (18) is a Lyapunov function for the hyperbolic system (25) and (26).

Note that the matrix inequality (22) considers, through the Lyapunov matrices $P_{1}$ and $P_{2}$, the coupling between the system's dynamics and the boundary conditions dynamics. As in Proposition 1, the strictly positive constant $\mu$ allows designing the convergence speed. Note that for a fixed $\mu,(22)$ becomes an LMI that can be solved using numerical procedures such as convex optimization algorithms.

Remark 3: The previous results (namely Proposition 1 and Theorem 1) extend to first order hyperbolic systems with both negative and positive convecting speeds $\left(\lambda_{1}<\ldots<\lambda_{m}<0<\lambda_{m+1}<\ldots<\lambda_{n}\right)$ by defining the state description $\xi=\left[\begin{array}{l}\xi_{-} \\ \xi_{+}\end{array}\right]$, where $\xi_{-} \in \mathbb{R}^{m}$ and $\xi_{+} \in \mathbb{R}^{n-m}$, and the variable transformation $\tilde{\xi}(x, t)=$ $\left(\begin{array}{c}\xi_{-}(1-x, t) \\ \xi_{+}(x, t)\end{array}\right)$.

\section{Boundary Observer for Quasi-Linear Hyper- bolic Systems}

In this section, under Assumption 2, we present sufficient conditions for exponential observer design for the quasi-linear hyperbolic system (1) and the boundary controls (4) and (5).

From Assumption 2, we know that there exist $\iota_{i}>$ $0, \forall i \in[1, \ldots, n]$ and a unique $C^{1}$ solution for (1) with boundary conditions (4) or (5) and initial condition (6) such that:

$$
\left\|\xi_{i}(., t)\right\|_{H^{1}} \leq \iota_{i}, \quad \forall t \geq 0, \quad \forall i \in[1, \ldots, n]
$$

where $\iota_{i} \in \mathbb{R}^{+}$. Moreover, from compact injection from $H^{1}(0,1)$ to $L^{\infty}(0,1)$, we know that there exists $C_{\xi}$ (which does not depend on the solution) such that:

$$
\begin{aligned}
& \left\|\xi_{i}(., t)\right\|_{L^{\infty}} \leq C_{\xi}\left\|\xi_{i}(., t)\right\|_{H^{1}} \leq C_{\xi} \iota_{i}=: \gamma_{i}, \\
& \forall t \geq 0, \quad \forall i \in[1, \ldots, n]
\end{aligned}
$$

Define $\Gamma_{\xi}=\operatorname{diag}\left(\gamma_{1}, \ldots, \gamma_{n}\right)$ and the non empty subset: 


$$
\Upsilon:=B\left(\gamma_{1}\right) \times \ldots \times B\left(\gamma_{n}\right) \subset \Theta
$$

As previously mentioned, the characteristic matrix $\Lambda(\xi)$ is continuous differentiable, implying that there exists a Lipschitz constant $\gamma_{\Lambda}>0$ such that:

$$
\left\|\partial_{\xi} \Lambda(\xi)\right\|<\gamma_{\Lambda}, \quad \forall \xi \in \Upsilon
$$

Also, from the continuity of $\Lambda$, the characteristic matrix can be bounded as follows:

$$
\left[\Lambda(\xi)-\Lambda_{\min }\right] \succeq 0 \quad \text { and } \quad\left[\Lambda_{\max }-\Lambda(\xi)\right] \succeq 0 \quad \forall \xi \in \Upsilon
$$

where $\Lambda_{\text {min }}, \Lambda_{\max } \in \mathbb{R}^{n \times n}$ are diagonal positive definite matrices which can be chosen, for example, as:

$$
\begin{aligned}
& \Lambda_{\text {min }}=\operatorname{diag}\left(\min _{\xi \in \Upsilon}\left(\lambda_{1}\right), . ., \min _{\xi \in \Upsilon}\left(\lambda_{n}\right)\right) \\
& \Lambda_{\text {max }}=\operatorname{diag}\left(\max _{\xi \in \Upsilon}\left(\lambda_{1}\right), \ldots, \max _{\xi \in \Upsilon}\left(\lambda_{n}\right)\right)
\end{aligned}
$$

Using the previous definitions and assumptions, Theorem 2 presents the sufficient conditions for the boundary observer design for (1) with boundary control (4).

Theorem 2. Consider the system (1) with static boundary conditions (4) and initial condition (6). Let $P \in$ $\mathbb{R}^{n \times n}$ be a diagonal positive definite matrix, $\mu>0$ be a constant and $L \in \mathbb{R}^{n \times n}$ be an observer gain such that:

$$
\begin{gathered}
e^{-\mu} \Lambda_{\min } P-L^{T} \Lambda_{\max } P L \succeq 0 \\
\Lambda_{\min }-\frac{3}{\mu} \gamma_{\Lambda} \Gamma_{\xi} \succ 0
\end{gathered}
$$

are satisfied, then:

$$
\partial_{t} \hat{\xi}(x, t)+\Lambda(\hat{\xi}(x, t)) \partial_{x} \hat{\xi}(x, t)=0
$$

$$
\hat{\xi}(0, t)=u_{c}(t)+L(\xi(1, t)-\hat{\xi}(1, t))
$$

is an exponential boundary observer for all twice continuously differentiable functions $\hat{\xi}^{0}:[0,1] \rightarrow \Upsilon$ satisfying the zero-order and one-order compatibility conditions.

Proof Defining $\varepsilon=\xi-\hat{\xi}$, the dynamics of the estimation error is given by:

$$
\partial_{t} \varepsilon(x, t)+\Lambda(\xi) \partial_{x} \varepsilon(x, t)+v_{e}=0
$$

with boundary condition

$$
\varepsilon(0, t)=-L \varepsilon(1, t)
$$

where $v_{e}=(\Lambda(\xi)-\Lambda(\hat{\xi})) \hat{\xi}_{x}$. From (35), it is possible to consider the term $v_{e}$ of (41) as a vanishing perturbation. This implies that as $\varepsilon \rightarrow 0$ in $L^{2},(\Lambda(\xi)-\Lambda(\hat{\xi})) \hat{\xi}_{x} \rightarrow$ 0 in $L^{2}$. From (32) and (35), this perturbation can be bounded for all $\xi:[0,1] \rightarrow \Upsilon$ as follows:

$$
\left\|v_{e}\right\|_{L^{\infty}}=\left\|(\Lambda(\xi)-\Lambda(\hat{\xi})) \hat{\xi}_{x}\right\|_{L^{\infty}} \leq \gamma_{\Lambda}\left\|\Gamma_{\xi} \varepsilon\right\|_{L^{\infty}}
$$

Given a diagonal positive definite matrix $P \in \mathbb{R}^{n \times n}$, consider (18) as a Lyapunov function candidate defined for all continuously differentiable functions $\varepsilon:[0,1] \rightarrow \Upsilon$. Computing the time derivative $\dot{V}$ of $V$ along the classical $C^{1}$-solutions of (41) with boundary conditions (42) yields to the following:

$$
\begin{aligned}
\dot{V}= & -\int_{0}^{1}\left(v_{e}^{T} P \varepsilon+\varepsilon^{T} P v_{e}\right) e^{-\mu x} d x \\
& -\mu \int_{0}^{1}\left(\varepsilon^{T} \Lambda(\xi) P \varepsilon\right) e^{-\mu x} d x \\
& +\int_{0}^{1}\left(\varepsilon^{T} \partial_{\xi} \Lambda(\xi) \xi_{x} P \varepsilon\right) e^{-\mu x} d x-\left.\left[e^{-\mu x} \varepsilon^{T} \Lambda(\xi) P \varepsilon\right]\right|_{0} ^{1}
\end{aligned}
$$

Using (32), (35), (37) and (43), the time derivative of the Lyapunov function can be bounded as: 


$$
\begin{aligned}
\dot{V} \leq & -\varepsilon(1)^{T}\left[e^{-\mu} \Lambda_{\min } P-L^{T} \Lambda_{\max } P L\right] \varepsilon(1) \\
& -\mu \int_{0}^{1}\left[\varepsilon^{T}\left(\Lambda_{\min } P-\frac{3}{\mu} \gamma_{\Lambda} \Gamma_{\xi} P\right) \varepsilon\right] e^{-\mu x} d x
\end{aligned}
$$

Conditions (38) imply that (45) is always negative. It can be easily shown from condition (38) that there exists a $\gamma_{\varepsilon}>0$ such that:

$$
\dot{V} \leq-\mu \gamma_{\varepsilon} V(\varepsilon)
$$

where $\gamma_{\varepsilon}$ could be for example the smallest eigenvalue of $\Lambda_{\min }-\frac{3}{\mu} \gamma_{\Lambda} \Gamma_{\xi}$. Therefore, the function (18) is a Lyapunov function for the hyperbolic system (25) with boundary conditions $(26)$ for all $\xi:[0,1] \rightarrow \Upsilon$.

Note that, unlike the linear hyperbolic case, not any $\mu>$ 0 ensures the stability of boundary observer for quasilinear hyperbolic systems. There is a minimum $\mu>0$ such that the condition (38) is satisfied. More precisely, Assumption 1 implies that $\Lambda_{\min } P \succ 0$. Therefore, there exists a finite $\mu>\mu_{\text {min }}$ and a small enough $L$ such that (38) holds and thus such that (39)-(40) is an exponential observer for the system (1) with boundary conditions (4). The $\mu_{\text {min }}$ can be determined for example as follows:

$$
\mu_{\min }=\max _{e i g}\left\{3 \gamma_{\Lambda} \Lambda_{\min }^{-1} \Gamma_{\xi}\right\}
$$

where $\max _{e i g}$ stands for maximal eigenvalue. Equation (47) implies that smaller values of $\mu$ are admissible when having large convecting speeds, and therefore faster observer convergence can be obtained.

In Theorem 3, sufficient conditions for the observer design with dynamic boundary conditions (5) are presented.

Theorem 3. Consider the system (1) with dynamic boundary condition (5) and initial conditions (6)-(7). Assume that there exist two diagonal positive definite matrices $P_{1}, P_{2} \in \mathbb{R}^{n \times n}$, a constant $\mu>0$ and an observer gain $L \in \mathbb{R}^{n \times n}$ such that:

$$
\left[\begin{array}{c|c}
A^{T} P_{1}+P_{1} A+C^{T} \Lambda_{\max } P_{2} C & -P_{1} L \\
+\mu \Lambda_{\min } P_{1}-3 \gamma_{\Lambda} \Gamma_{\xi} P_{1} & \\
\hline-L^{T} P_{1} & -e^{-\mu} \Lambda_{\min } P_{2}
\end{array}\right] \preceq 0
$$

are satisfied, then:

$$
\partial_{t} \hat{\xi}(x, t)+\Lambda \partial_{x} \hat{\xi}(x, t)=0
$$

$$
\begin{aligned}
& \dot{\hat{X}}_{c}=A_{c} \hat{X}_{c}+B_{c} u(t)+L(\xi(1, t)-\hat{\xi}(1, t)) \\
& \hat{\xi}(0, t)=C_{c} \hat{X}_{c}+D_{c} u(t)
\end{aligned}
$$

is an exponential boundary observer for all continuously differentiable functions $\hat{\xi}^{0}:[0,1] \rightarrow \Upsilon$ and for all $\hat{X}_{c}^{0} \in \mathbb{R}^{n}$ satisfying the zero-order and one-order compatibility conditions.

Proof. Define the dynamics of the estimation error as in (41) with boundary conditions (26). Using the same vanishing perturbation approach as in the proof of Theorem 2 and the Lyapunov function candidate (28) defined for all continuously differentiable functions $\varepsilon:[0,1] \rightarrow \Upsilon$ gives:

$$
\begin{aligned}
& \dot{V}=\varepsilon^{T}\left(A^{T} P_{1}+P_{1} A\right) \varepsilon-\varepsilon(1)^{T} L^{T} P_{1} \varepsilon-\varepsilon^{T} P_{1} L \varepsilon(1) \\
& -\left.\left[e^{-\mu x} \varepsilon^{T} \Lambda(\xi) P_{2} \varepsilon\right]\right|_{0} ^{1}-\mu \int_{0}^{1}\left(\varepsilon^{T} \Lambda(\xi) P_{2} \varepsilon\right) e^{-\mu x} d x \\
& +\int_{0}^{1}\left(\varepsilon^{T} \partial_{\xi} \Lambda(\xi) \xi_{x} P_{2} \varepsilon\right) e^{-\mu x} d x \\
& -\int_{0}^{1}\left(v_{e}^{T} P_{2} \varepsilon+\varepsilon^{T} P_{2} v_{e}\right) e^{-\mu x} d x
\end{aligned}
$$

After expanding (51), we obtain the following: 


$$
\begin{aligned}
\dot{V}= & -\mu \int_{0}^{1}\left(\varepsilon^{T} \Lambda(\xi) P_{2} \varepsilon\right) e^{-\mu x} d x \\
& +\int_{0}^{1}\left(\varepsilon^{T} \partial_{\xi} \Lambda(\xi) \xi_{x} P_{2} \varepsilon\right) e^{-\mu x} d x \\
& -\int_{0}^{1}\left(v_{e}^{T} P_{2} \varepsilon+\varepsilon^{T} P_{2} v_{e}\right) e^{-\mu x} d x+\left[\begin{array}{c}
\varepsilon \\
\varepsilon(1)
\end{array}\right] \\
& \times\left[\begin{array}{c|c}
A^{T} P_{1}+P_{1} A+ & -P_{1} L \\
C^{T} \Lambda(\xi(0)) P_{2} C & \\
\hline-L^{T} P_{1} & -e^{-\mu} \Lambda(\xi(1)) P_{2}
\end{array}\right]\left[\begin{array}{c}
\varepsilon \\
\varepsilon(1)
\end{array}\right]
\end{aligned}
$$

With the Schur complement, it can be easily shown that for all $\xi:[0,1] \rightarrow \Upsilon$ :

$$
\begin{gathered}
{\left[\begin{array}{cc}
A^{T} P_{1}+P_{1} A+C^{T} \Lambda_{\max } P_{2} C & -P_{1} L \\
-L^{T} P_{1} & -e^{-\mu} \Lambda_{\min } P_{2}
\end{array}\right]} \\
-\left[\begin{array}{cc}
A^{T} P_{1}+P_{1} A & -P_{1} L \\
+C^{T} \Lambda(\xi(0)) P_{2} C & \\
\hline-L^{T} P_{1} & -e^{-\mu} \Lambda(\xi(1)) P_{2}
\end{array}\right] \succ 0
\end{gathered}
$$

Using (32), (35), (37) and (43), we can bound the Lyapunov function time derivative as follows:

$$
\begin{aligned}
& \dot{V} \leq-\mu \int_{0}^{1}\left[\left(\varepsilon^{T}\left(\Lambda_{\min } P_{2}-\frac{3}{\mu} \gamma_{\Lambda} \Gamma_{\xi} P_{2}\right) \varepsilon\right)\right] e^{-\mu x} d x \\
& -\mu \varepsilon^{T}\left[\Lambda_{\min } P_{1}-\frac{3}{\mu} \gamma_{\Lambda} \Gamma_{\xi} P_{1}\right] \varepsilon+\left[\begin{array}{c}
\varepsilon \\
\varepsilon(1)
\end{array}\right]^{T} \\
& \times\left[\begin{array}{c|c}
A^{T} P_{1}+P_{1} A & \\
+C^{T} \Lambda_{\max } P_{2} C & -P_{1} L \\
+\mu \Lambda_{\min } P_{1}-3 \gamma_{\Lambda} \Gamma_{\xi} P_{1} & \\
\hline-L^{T} P_{1} & -e^{-\mu} \Lambda_{\min } P_{2}
\end{array}\right]\left[\begin{array}{c}
\varepsilon \\
\varepsilon(1)
\end{array}\right]
\end{aligned}
$$

The matrix inequalities in (48) imply that the third term of (54) is always negative or zero. Thus, as detailed in the proof of Theorem 2, it can be easily shown that:

$$
\dot{V} \leq-\mu \gamma_{\varepsilon} V\left(\varepsilon, \varepsilon_{c}\right)
$$

Therefore, for a suitable $\gamma_{\varepsilon}>0$, the function (28) is a Lyapunov function for the hyperbolic system (41) and (26) for all $\xi:[0,1] \rightarrow \Upsilon$.

Like in Theorem 2, the value of $\mu$ cannot be any positive constant as it has some restrictions given by the second line of (48). To perform the boundary observer design, first a $\mu$ that satisfies the second line of (48) is computed. Then, this value of $\mu$ is employed to compute $L, P_{1}$ and $P_{2}$, solution of (48).

\section{Application to an Incompressible Flow Speed Estimation}

In this section, we model an inviscid incompressible flow inside a pipe of constant cross section using the Burger's equation (quasi-linear hyperbolic system). We solve the state estimation problem using the boundary observer design presented in the previous sections. We design two boundary observers: one to observe the system with static boundary conditions (Theorem 2) and a second one to observe the system with dynamic boundary conditions (Theorem 3).

Figure 1 presents the schematic of the estimation problem that we propose to illustrate the main results of this work. As depicted, there are two tubes in parallel which share a common input boundary system denoted as $\Sigma$. The variables $w_{1}$ and $w_{2}$ describe the fluid speed distribution of each tube, while $u_{c 1}$ and $u_{c 2}$ are the system $\Sigma$ control inputs. The system $\Sigma$ is considered to be a static system as well as a dynamic one with the purpose of applying the results of Theorem 2 and 3, respectively. In practice, for example, the measurements at the outputs $\left(w_{1}(1, t)\right.$ and $\left.w_{2}(1, t)\right)$ could be obtained directly by flow rate sensors and the actuation at the left boundaries could be performed with ventilators.

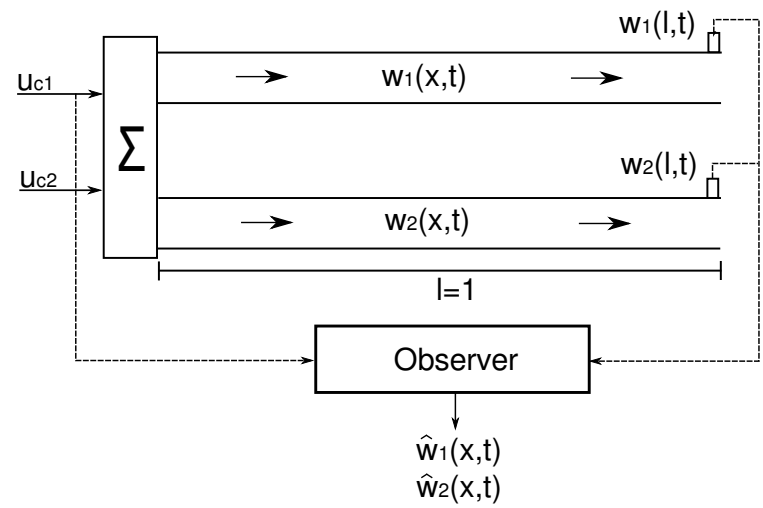

Fig. 1. Schematic of the estimation problem 
To model the flow inside the pipes, let us consider the one-dimensional inviscid Burger's equation which is the result of dropping the pressure term in the incompressible Navier-Stockes equations [18]:

$$
\partial_{t} w(x, t)+\Lambda(w(x, t)) \partial_{x} w(x, t)=0
$$

where $w=\left[w_{1}, w_{2}\right]^{T}$ and

$$
\Lambda(w)=\left[\begin{array}{cc}
w_{1}(x, t) & 0 \\
0 & w_{2}(x, t)
\end{array}\right]
$$

Let us assume that (57) satisfies Assumption 1. Define the following change of coordinates:

$$
\xi_{1}=w_{1}-\tilde{w}_{1}, \quad \xi_{2}=w_{2}-\tilde{w}_{2}
$$

where $\tilde{w}=\left[\tilde{w}_{1}, \tilde{w}_{2}\right]^{T}$ is an arbitrary reference. With these new coordinates $\left(\xi_{1}, \xi_{2}\right)$, system (56) can be rewritten in the quasi-linear hyperbolic form (1) as follows:

$\partial_{t}\left(\begin{array}{l}\xi_{1} \\ \xi_{2}\end{array}\right)+\left[\begin{array}{cc}\xi_{1}+\tilde{w}_{1} & 0 \\ 0 & \xi_{2}+\tilde{w}_{2}\end{array}\right] \partial_{x}\left(\begin{array}{l}\xi_{1} \\ \xi_{2}\end{array}\right)=\left(\begin{array}{l}0 \\ 0\end{array}\right)$

Consider the dynamic boundary conditions according to (5) with the respective matrices given by:

$$
\begin{gathered}
A=\left[\begin{array}{cc}
-11 & 4 \\
5 & -8
\end{array}\right], \quad B=\left[\begin{array}{cc}
8 & 6 \\
4 & 10
\end{array}\right], \\
C=I^{2}, \quad D=0^{2 \times 2}
\end{gathered}
$$

and also consider the static boundary conditions given by:

$$
\xi(0, t)=-C A^{-1} B u_{c}(t)
$$

Note that due to (60), there is a coupling between the control inputs and the boundary conditions. The static boundary conditions (61) are chosen in such a way, with the purpose of obtaining flow speeds of similar magnitudes as in the dynamic case and perform a comparison using the same $u_{c}$ for both cases. We require, for the boundary observer design, the definition of the subset $\Upsilon$ as well as a Lipschitz constant according to (34) and (35), respectively. We define $\tilde{w}=[7,5]^{T}$. To build the subset $\Upsilon$, let us first establish the allowable flow speeds variation as $+/-0.6$ which gives:

$$
\begin{aligned}
& w_{1} \in[6.4,7.6] \rightarrow \xi_{1} \in[-0.6,0.6] \\
& w_{2} \in[4.4,5.6] \rightarrow \xi_{2} \in[-0.6,0.6]
\end{aligned}
$$

From (34), the subset $\Upsilon$ can be defined as:

$$
\Upsilon:=\{B(0.6) \times B(0.6)\}
$$

Due to (57), the Lipschitz constant is $\gamma_{\Lambda}=1$. From (62), the characteristic matrix $\Lambda(\xi)$ can be bounded according to (37) with:

$$
\Lambda_{\min }=\left[\begin{array}{cc}
6.4 & 0 \\
0 & 4.4
\end{array}\right], \Lambda_{\max }=\left[\begin{array}{cc}
7.6 & 0 \\
0 & 5.6
\end{array}\right]
$$

For the boundary observer evaluation, the system control input $u_{c}$ varies with respect to time according to Figure 2. However, the variation magnitude is constrained to ensure that $\xi:[0,1] \rightarrow \Upsilon$. The same $u_{c}$ is used for the dynamics and the static boundary conditions.

\subsection{Static Boundary Observer}

In this subsection, we design a boundary observer for system (59) with static boundary conditions (61). From the results of Theorem 2, it can be easily found that for any $\mu>0.48$, the second inequality of (38) holds. Considering

$$
\begin{gathered}
P=\left[\begin{array}{lll}
1 & 0 & 0 \\
0 & 1 & 0 \\
0 & 0 & 1
\end{array}\right], \quad L_{1}=\left[\begin{array}{cc}
0.3 & -0.1 \\
-0.2 & 0.3
\end{array}\right], \\
\mu=0.6
\end{gathered}
$$



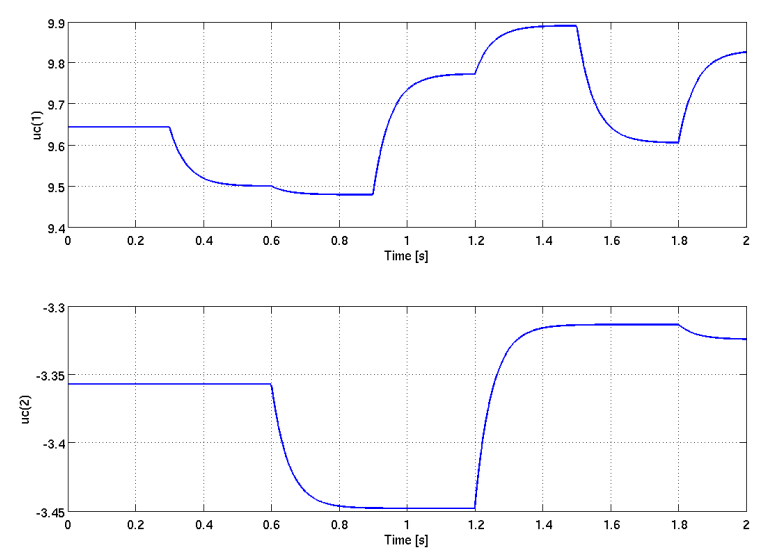

Fig. 2. Boundary system inputs

which satisfy the conditions of (38), we obtain an exponential boundary observer for (59) with boundary conditions (61) for all $\xi:[0,1] \rightarrow \Upsilon$. Note that the observer gain $L_{1}$ has been chosen to introduce a state coupling in the observer design. In order to evaluate the effectiveness of the observer, the Burger equations are simulated using a MacCormack numerical method combined with a time varying diminishing (TVD) scheme. Figures 3 and 4 show the flow speed estimation error profile for each tube using the static boundary observer (39)-(40) and observer initial conditions $[-0.5,0.5]^{T} \in \Upsilon$.

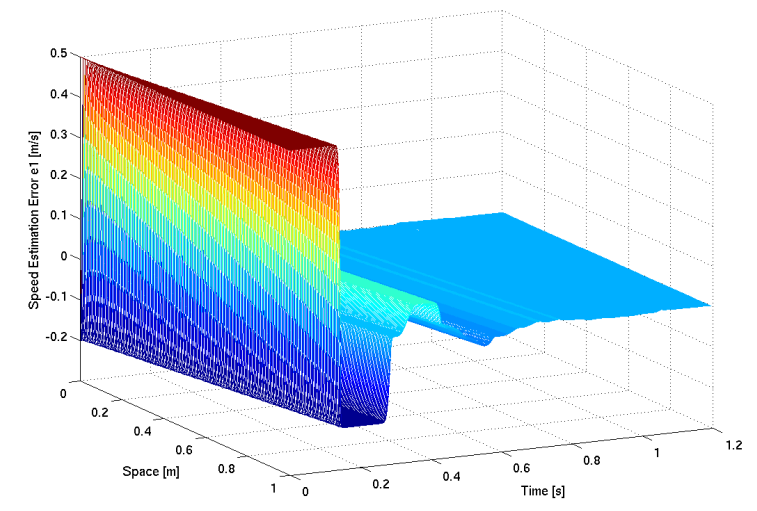

Fig. 3. Profile of the flow speed estimator error of Tube 1 using the boundary observer (39)-(40)

As depicted in Figures 3 and 4, the estimation error converges to zero in finite time as expected from the results of Theorem 2 . The simulation results illustrate the effectiveness of the proposed sufficient conditions for exponential boundary observer design. Figure 5 depicts the observer input $v(t)=L(\xi(1, t)-\hat{\xi}(1, t))$ and 6 the Lyapunov function (18).

\subsection{Dynamic Boundary Observer}

In this subsection, we design a boundary observer for system (59) with dynamic boundary conditions (5). Once

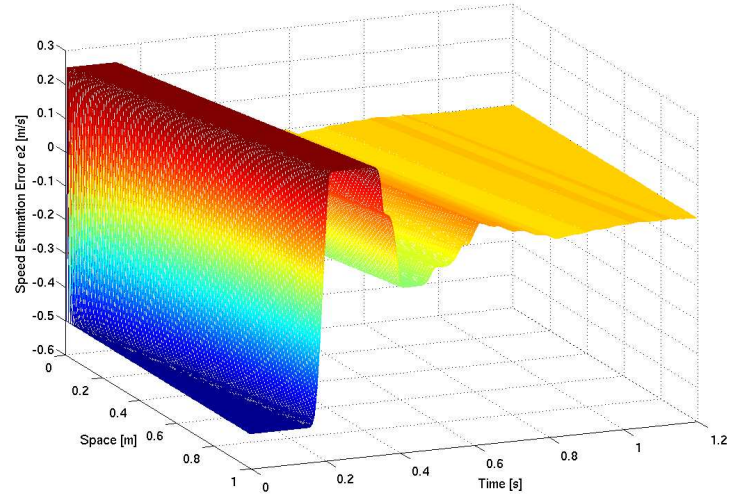

Fig. 4. Profile of the flow speed estimator error of Tube 2 using the boundary observer (39)-(40)

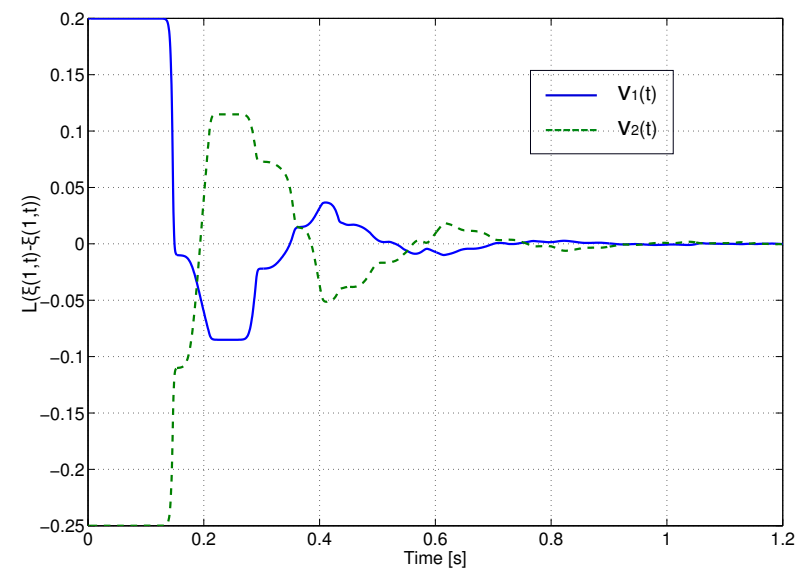

Fig. 5. The time evolution of the observer input $v(t)$.

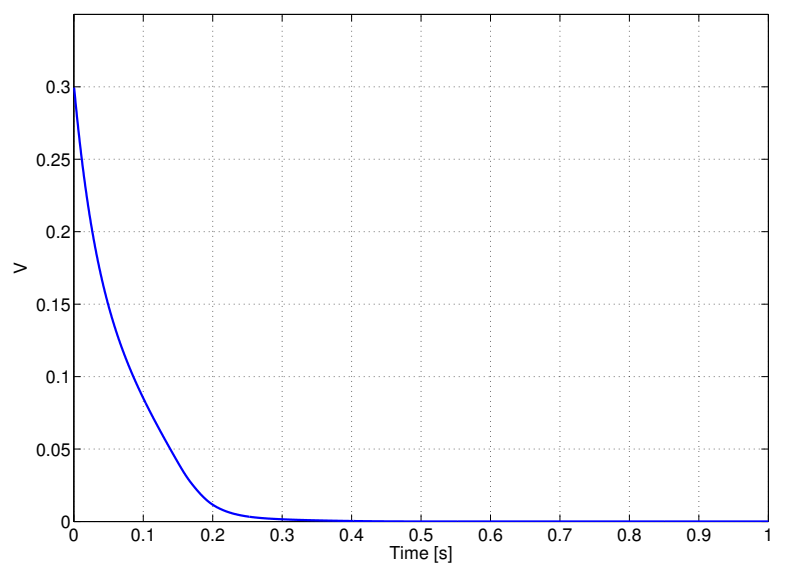

Fig. 6. Time evolution of the Lyapunov function for the static boundary conditions

again, we consider the initial conditions in $\Upsilon$ given by (63) and the dynamic boundary conditions defined in (60). We can easily verify that $\mu>0.48$ satisfies the second inequality of (48). Choosing $\mu=0.5$ in (48) and considering $P_{1}=P_{2}$ yields an LMI for the unknown variables $L$ and $P_{1}$. To find a suitable observer gain, a 
convex optimization algorithm is used to find $P_{1}$ as well as $L$ while maximizing $\operatorname{Trace}(L)$ (other criteria may be used). The results obtained are the following:

$$
L_{2}=\left[\begin{array}{cc}
2.2556 & -1.0795 \\
-1.8259 & 1.2341
\end{array}\right]
$$

with the respective diagonal positive definite matrix associated with the Lyapunov function (18):

$$
P_{1}=P_{2}=\left[\begin{array}{cc}
0.2555 & 0 \\
0 & 0.3433
\end{array}\right]
$$

Figures 7 and 8 show the results obtained with the observer (49) - (50) with gain (66) and the observer initial conditions $[-0.5,0.5]^{T} \in \Upsilon$.

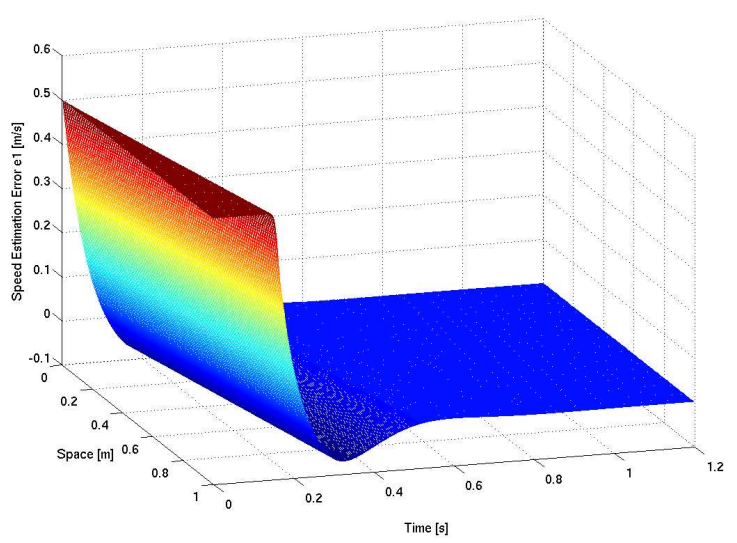

Fig. 7. Profile of the flow speed estimator error of Tube 1 using the boundary observer (49)-(50)

As depicted in Figures 7, and 8, the estimation error converges to zero in finite time as expected from Theorem 3. The simulation results illustrate the effectiveness of the proposed sufficient conditions for exponential boundary observer design. Figure 9 shows the observer input $v(t)=L(\xi(1, t)-\hat{\xi}(1, t))$ and 10 the Lyapunov function (28).

\section{Conclusion}

In this paper, we designed boundary observers for linear and quasi-linear hyperbolic systems with $n$ rightward convective transport PDEs. We obtained some sufficient

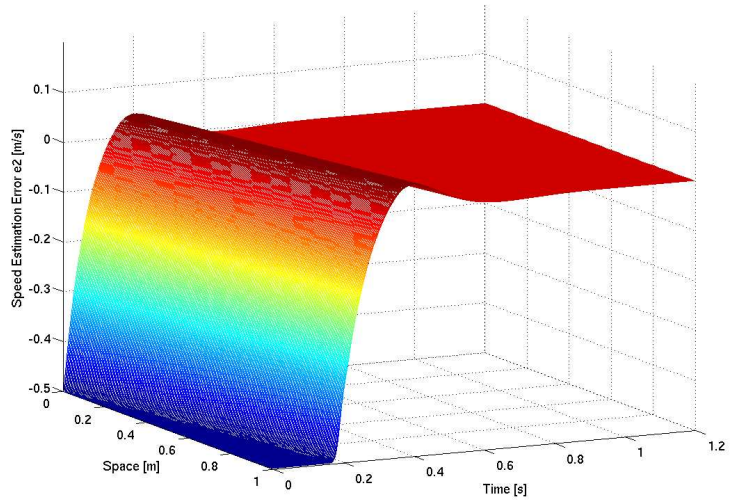

Fig. 8. Profile of the flow speed estimator error of Tube 2 using the boundary observer (49)-(50)

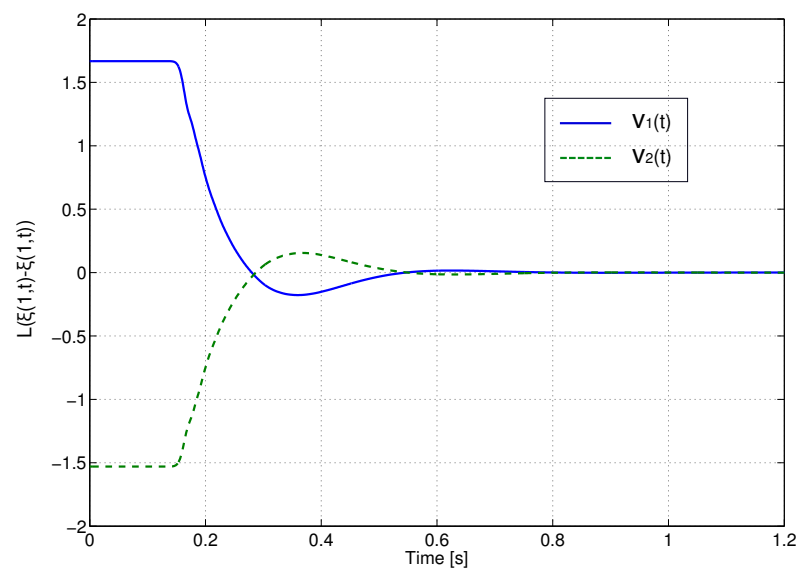

Fig. 9. The time evolution of the observer input $v(t)$.

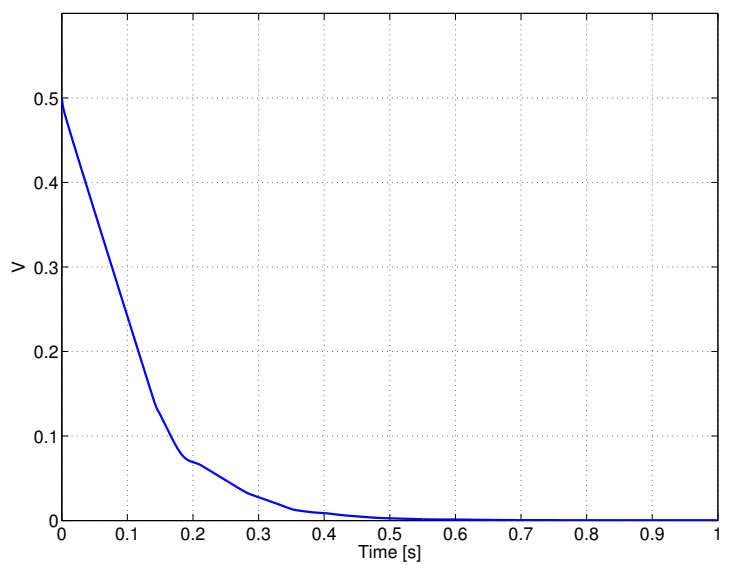

Fig. 10. Time evolution of the Lyapunov function for the dynamic boundary conditions

conditions for exponential boundary observer design for linear and quasi-linear first order hyperbolic PDE with static and dynamic boundary control. We have demonstrated the exponential convergence of the estimation error by means of a Lyapunov function formulation. A simulation example has shown the effectiveness of our 
results for a quasi-linear hyperbolic system with static and dynamic boundary control.

This work has many applications in different systems governed by hyperbolic PDE. However, many questions are still open. In particular, the boundary observer design of quasilinear first order hyperbolic systems with dynamics boundary conditions and $n$ rightward and $m$ leftward convective transport PDEs seems to be a challenging issue. The use of the Lyapunov function formulated in [8] may be a good approach to address this issue. The derivation of boundary observers for hyperbolic systems with non-linear dynamic boundary control is also another interesting subject to investigate. A polytopic extension of Theorems 1 and 3 seems to be a natural extension of this work to address this issue.

\section{References}

[1] O. Aamo, J. Salvesen, and B. Foss. Observer design using boundary injections for pipelines monitoring and leak detection. International symposium on advance control of chemical processes, pages 53-58, 2006.

[2] S. Alinhac. Hyperbolic Partial Differential Equations. Springer, London, 2009.

[3] G. Bastin, J-M. Coron, and B. d'Andréa Novel. Using hyperbolic systems of balance laws for modelling, control and stability analysis of physical networks. Lecture notes for the Pre-Congress Workshop on Complex Embedded and Networked Control Systems, 17th IFAC World Congress, Seoul, Korea, 2008.

[4] F. Castillo, E. Witrant, and L. Dugard. Contrôle de température dans un flux de Poiseuille. Proceedings of the IEEE Conférence Internationale Francophone d'Automatique, Grenoble, France, 2012.

[5] F. Castillo, E. Witrant, C. Prieur, and L. Dugard. Dynamic boundary stabilization of linear and quasi-linear hyperbolic systems. Proceedings of the 51st IEEE Conference on Decision and Control, Maui, Hawai, pages 2952-2957, 2012.

[6] J-M. Coron, G. Bastin, and B. d'Andréa Novel. Dissipative boundary conditions for one-dimensional nonlinear hyperbolic systems. SIAM J. Control Optim, 47:1460-1498, 2008.

[7] J-M. Coron, B. d'Andréa Novel, and G. Bastin. A strict Lyapunov function for boundary control of hyperbolic systems of conservation laws. IEEE Transactions on Automatic control, 52:2-11, 2007.

[8] A. Diagne, G. Bastin, and J-M. Coron. Lyapunov exponential stability of 1-D linear hyperbolic systems of balance laws. Automatica, 48:109-114, 2012.

[9] F.M. Haut, G. Leugering, and T.I. Seidman. Modeling and analysis of modal switching in networked transport systems. Applied Mathematics and Optimization, 59(2):275-292, 2009.

[10] V. Kato. The Cauchy problem for quasi-linear symmetric hyperbolic systems. Arch. Rational Mech. Anal., 58:181-205, 1985.

[11] M. Krstic and A. Smyshlyaev. Backstepping boundary control for first-order hyperbolic PDEs and application to systems with actuator and sensor delays. Systems and Control Letters, 57(9):750-758, 2008.
[12] M. Krstic and A. Smyshlyaev. Boundary Control of PDEs: A Course on Backstepping Designs. Society for Industrial and Applied Mathematics, Philadelphia, USA, 2008.

[13] T.T. Li. Controllability and observability for quasilinear hyperbolic systems. High Education Press, Beijing, 3, 2012.

[14] F. Di Meglio, G.O. Kaasa, N. Petit, and V. Alstad. Slugging in multiphase flow as a mixed initial-boundary value problem for a quasilinear hyperbolic system. Proceedings of the American Control Conference, San Francisco, USA, pages 3589-3596, 2011.

[15] C. Prieur, J. Winkin, and G. Bastin. Robust boundary control of systems of conservation laws. Mathematics of Control, Signals, and Systems, 20(2):173-197, 2008.

[16] V. Dos Santos, G. Bastin, J-M. Coron, and B. d'Andréa Novel. Boundary control with integral action for hyperbolic systems of conservation laws: stability and experiments. Automatica, 44(1):1310-1318, 2008.

[17] V. Dos Santos and C. Prieur. Boundary control of open channels with numerical and experimental validations. IEEE Transactions on Control Systems Technology, 16(6):12521264, 2008.

[18] J. Tannehill, D. Anderson, and R. Pletcher. Computational Fluid Mechanics and Heat Transfer. Taylor and Francis, Washington DC, USA, 1997.

[19] M. Tucsnak and G. Weiss. Observation and control for operator semigroups. Birkhauser Advanced Texts, Germany, 2009.

[20] F. Vazquez, M. Krstic, and J-M. Coron. Backstepping boundary stabilization and state estimation of a $2 \times 2$ linear hyperbolic system. Proceedings of the 50th IEEE Conference on Decision and Control and European Control Conference, Orlando, FL, USA, pages 4937-4942, 2011.

[21] E. Witrant, A. D'Innocenzo, G. Sandou, F. Santucci, M. D. Di Benedetto, A. J. Isaksson, K. H. Johansson, S.I. Niculescu, S. Olaru, E. Serra, S. Tennina, and U. Tiberi. Wireless ventilation control for large-scale systems: the mining industrial case. International Journal of Robust and Nonlinear Control, 20:226-251, Jan. 2010. 